\title{
JMJD3 is a histone H3K27 demethylase
}

\author{
Yang Xiang ${ }^{1}$, Ziqi Zhu ${ }^{1}$, Gang Han ${ }^{1}$, Hanqing Lin ${ }^{1}$, Longyong $\mathrm{Xu}^{1}$, Charlie Degui Chen ${ }^{1}$ \\ ${ }^{1}$ State Key Laboratory of Molecular Biology, Institute of Biochemistry and Cell Biology, Shanghai Institutes for Biological Sciences, \\ Chinese Academy of Sciences, Shanghai 200031, China
}

\begin{abstract}
Histone methylation is an important epigenetic phenomenon that participates in a diverse array of cellular processes and has been found to be associated with cancer. Recent identification of several histone demethylases has proved that histone methylation is a reversible process. Through a candidate approach, we have biochemically identified JMJD3 as an H3K27 demethylase. Transfection of JMJD3 into HeLa cells caused a specific reduction of trimethyl H3K27, but had no effect on di- and monomethyl H3K27, or histone lysine methylations on H3K4 and H3K9. The enzymatic activity requires the JmjC domain and the conserved histidine that has been suggested to be important for a cofactor binding. In vitro biochemical experiments demonstrated that JMJD3 directly catalyzes the demethylation. In addition, we found that JMJD3 is upregulated in prostate cancer, and its expression is higher in metastatic prostate cancer. Thus, we identified JMJD3 as a demethylase capable of removing the trimethyl group from histone H3 lysine 27 and upregulated in prostate cancer.
\end{abstract}

Keywords: histone methylation, demethylase, JMJD3, H3K27, prostate cancer

Cell Research (2007) 17:850-857. doi: 10.1038/cr.2007.83; published online 9 October 2007

\section{Introduction}

Histone methylation is an important epigenetic phenomenon that participates in a diverse array of cellular processes [1]. Histone methylation can occur on either arginine or lysine residues [2]. Each lysine residue can undergo three stages of methylation, having either one (mono), two (di), or three (tri) methyl groups covalently bonded to the amine group of the lysine side chain, and arginine can be monoor dimethylated [3]. Depending on specific residues and methylation status, methylation can activate or repress transcription. In general, lysine methylation at $\mathrm{H} 3 \mathrm{~K} 9$, $\mathrm{H} 3 \mathrm{~K} 27$, and $\mathrm{H} 4 \mathrm{~K} 20$ is associated with transcriptional repression, whereas methylation at $\mathrm{H} 3 \mathrm{~K} 4, \mathrm{H} 3 \mathrm{~K} 36$, and H3K79 is associated with transcriptional activation. While $\mathrm{H} 3 \mathrm{~K} 4$ methylation is associated with euchromatin function,

Correspondence: Charlie Degui Chen

Tel: +86-021-54921148; Fax: +86-021-54921148

E-mail: cdchen@sibs.ac.cn

Received 4 September 2007; revised 12 September; accepted 13 September; published online 9 October 2007 methylation of $\mathrm{H} 3 \mathrm{~K} 9$ is involved in heterochromatin formation [4]. Furthermore, methylation of H3K27 is involved in X-chromosome inactivation [5].

Histone methylation had been considered a stable modification, but recent studies have proved otherwise [6-16]. LSD1 is the first histone demethylase identified and uses an amine oxidase reaction to catalyze the removal of methylation [8]. LSD1 was shown to be a demethylase for $\mathrm{H} 3 \mathrm{~K} 4$ or $\mathrm{H} 3 \mathrm{~K} 9$ dependent upon associated factors [17, 18]. Subsequently, a JmjC domain containing protein was shown to possess histone demethylase activity and the $\mathrm{JmjC}$ domain was identified as a demethylase signature motif [9]. This family of enzymes catalyzes the removal of methylation by a hydroxylation reaction and required iron and $\alpha$-ketoglutarate as cofactors. Based on this demethylase signature motif, several proteins were identified to be histone lysine demethylases [6, 7, 10-16].

Prostate cancer is the most common non-skin cancer and the second leading cause of cancer in America. A mass screening in Changchun city reveals that the detection rate of prostate cancer was $0.74 \%$ in men with more than 50 years of age, indicating that the prevalence of prostate can- 
cer is high in Chinese population [19]. Histone methylation has been suggested to be associated with prostate cancer. For example, it was demonstrated that histone methylations and acetylations can predict the risk of prostate cancer recurrence [20]. Also, EZH2, an H3K27 methyltransferase, was shown to be involved in the progression of prostate cancer [21]. In addition, two of the identified histone demethylases LSD1 and JHDM2A were demonstrated to potentiate androgen receptor (AR)-dependent transcription through removal of $\mathrm{H} 3 \mathrm{~K} 9$ methylation $[11,17]$. AR, a member of the nuclear receptor superfamily, plays a key role in prostate cancer development and progression [22].

To identify novel histone demethylases, we selected a handful of JmjC domain containing proteins and examined their possible demethylase activities against H3K27. Through biochemical approaches, we identified JMJD3 as a histone demethylase that can specifically catalyze the removal of the trimethyl group from the H3K27 lysine residue. We also demonstrated that JMJD3 is upregulated in prostate cancer.

\section{Materials and Methods}

\section{Reagents}

Sources of the antibodies used are as follows: JMJD3 (Abgene AP1022b), H3 trimethyl-K4 (Abcam 8580), H3 monomethyl-K9 (Abcam 9045), H3 dimethyl-K9 (Upstate 07-212), H3 trimethylK9 (Abcam 8898), H3 monomethyl-K27 (Upstate 07-448), H3 dimethyl-K27 (Upstate 07-452), H3 trimethyl-K27 (Dr Jenuwein and Upstate 07-449), H3 (abcam1791), Myc (Calbiochem OP10) and (Sigma c-3956), Goat anti-Rabbit IgG (Jackson), Goat anti-Mouse IgG (Jackson), Donkey anti-Rabbit (Molecular Probes Alexa 594) and Goat anti-Mouse (Molecular Probes Alexa 488).

\section{Plasmid construction}

JMJD3 cDNA (KIAA0346) was kindly provided by Kazusa DNA Research Institute. pcDNA3.1-JMJD3-MYC-His was generated by the following two steps. A fragment covering the ORF from nucleotide (nt) 15 to nt 4386 was digested by BamHI and VspI. A PCR fragment covering nt 4387 to nt 5037 was digested with $V s p$ I and XhoI. Both fragments were ligated into BamHI and XhoI digested pcDNA3.1(-)/MYC-His by three-way ligation. This vector was inserted by 1-15 bp of JMJD3 with a Kozak sequence using NheI and BamHI. The JmjC domain deletion was generated by threeway ligation. PCR fragments from nt 2050 to nt 3987 and from nt 4584 to the end were digested with EcoRI-ClaI and ClaI-XhoI, and ligated to $E c o$ RI-XhoI digested pcDNA3-JMJD3. The primers for the fragment of nt 2050 to nt 3987 are: gccgaattcAAGATC CTACCTGATG and gacaaatcGATGTTGGTGCCAAAC. The primers for the fragment from nt 4584 to the end are: gacatcgatTTGTT CAAGATGATCAAG and atactcgagTCGCGACGTGCTGGC TGGGG. H1390A mutation was generated by the following two steps. First a PCR product from nt 3775 to the end was ligated to T-vector and the mutation was introduced by a site-directed mutagenesis. The fragment with H1390A mutation was swapped back to pcDNA3JMJD3. The primers for PCR are: ACTCGGCAGATCTGGC CTTGTGA and atactcgagTCGCGACGTGCTGGCTGGGG. The site-directed mutagenesis primers are: CAGCCGAACGCCAGGCG CTCAGGAGAATAACAACTTCTGC and GCAGAAGTTGTTAT TCTCCTGAGCGCCTGGCGTTCG GCTG. A DNA fragment covering 3991 to 4506 of JMJD3 cDNA was generated by PCR using a primer pair: ATAGGATCCTTGTCTGATGCTAAGCGGTG and ATACTCGAGGACG TTCTTCACCTCATTCC. The PCR product was digested with BamHI and XhoI, and ligated to Bam HI and XhoI digested pGEX4T1 to generate GST-JmjC domain of JMJD3 that contains only the JmjC domain.

\section{Transfection and western blotting}

HeLa cells were grown in DMEM containing 10\% FBS. Transfection was carried out using Lipofectamine 2000 (Invitrogen). Tissues were obtained from the radical prostatectomy performed at Sixth People Hospital of Shanghai with prior approval from the Institutional Review Board. Proteins from the cell or tissue lysates were separated on SDS-PAGE gel and western blot analysis was carried out using specific antibodies.

\section{Immunocytochemistry}

HeLa cells were plated on glass coverslips and transfected next day. $72 \mathrm{~h}$ later, the cells were fixed in $4 \%$ paraformaldehyde and permeabilized with cold PBS containing $0.2 \%$ Triton X-100. After blocking, the cells were incubated with primary antibodies, washed with secondary antibodies, stained with DAPI, and mounted before viewing.

Expression and purification of GST-JmjC domain of JMJD3 GST-JmjC domain of JMJD3 in Rosetta E. coli was cultured at $37{ }^{\circ} \mathrm{C}$ at 250 r.p.m.. Expression of the fusion protein was induced by IPTG $\mathrm{O} / \mathrm{N}$ at $16{ }^{\circ} \mathrm{C}$ when OD reached $0.5-0.6$. Bacterial was collected, resuspended in $1 \times$ PBS (PH 7.4), and sonicated 25 times at $200 \mathrm{~W}$ (sonicated $2 \mathrm{~s}$ and paused $15 \mathrm{~s}$ ). Supernatant was loaded into equilibrated Glutathione Sepharose column, incubated for 30 min at $4{ }^{\circ} \mathrm{C}$ and washed with $1 \times$ PBS for three times. The recombinant fusion protein was eluted by $20 \mathrm{mM}$ glutathione in $50 \mathrm{mM}$ Tris-HCl (pH 8.0).

\section{Demethylation assay}

Bulk calf thymus type II-A histone proteins (Sigma \#H9250) were incubated with the purified fusion protein of GST-JmjC domain of JMJD3 in demethylation buffer $(20 \mathrm{mM}$ Tris- $\mathrm{HCl}$ pH 7.3, $150 \mathrm{mM}$ $\mathrm{NaCl}, 50 \mu \mathrm{M}\left(\mathrm{NH}_{4}\right)_{2} \mathrm{Fe}\left(\mathrm{SO}_{4}\right)_{2}+6\left(\mathrm{H}_{2} \mathrm{O}\right), 1 \mathrm{mM} \alpha$-ketoglutarate, and 2 $\mathrm{mM}$ ascorbic acid) for $3 \mathrm{~h}$ at $37^{\circ} \mathrm{C}$. A total of 5-10 $\mu \mathrm{g}$ of the fusion protein and 2.5-5 $\mu \mathrm{g}$ of bulk histones were reacted in a total volume of $100 \mu \mathrm{l}$ of reaction $(5 \mu \mathrm{g}$ of the fusion protein and $5 \mu \mathrm{g}$ of bulk histones for Figure 4B, $10 \mu \mathrm{g}$ of the fusion protein and $2.5 \mu \mathrm{g}$ of bulk histones for Figure 4C). The reaction was stopped with SDS loading buffer and western blot analysis was performed.

\section{Results}

\section{JMJD3 demethylates H3K27 in vivo}

Since JmjC domain containing proteins have been demonstrated as demethylases for H3K9 and H3K36 [7, 9-11], we have been interested in the identification of JmjC domain containing proteins as demethylases for other lysine residues such as $\mathrm{H} 3 \mathrm{~K} 4$ and $\mathrm{H} 3 \mathrm{~K} 27$. Bioinformatic 
approaches revealed that 30 human genes contain the JmjC domain, which has been identified as the catalytic domain for one class of histone lysine demethylases $[9,11]$. These 30 genes can be clustered into seven groups: JHDM1, JARID, JMJD1 (JHDM2), JMJD2 (JHDM3), UTX/UTY, PHF2/PHF8, and JmjC domain only [23]. Genes in groups of JHDM1, JMJD1, and JMJD2 have been biochemically demonstrated to possess histone demethylase activity for histone $\mathrm{H} 3$ and they can specifically demethylate $\mathrm{H} 3 \mathrm{~K} 9$ or H3K36 [7, 9-11]. Through a candidate approach, we selected a handful of JmjC domain containing proteins and examined their possible demethylase activities against $\mathrm{H} 3 \mathrm{~K} 4$ and H3K27 using currently available antibodies. During this process, we discovered that JARID1B (also named PLU-1), a member of the JARID family, is a histone demethylase specific for H3K4 [24], which is consistent with a recent report [15]. We also examined whether JMJD3, a member of the UTX/UTY family, is a demethylase for $\mathrm{H} 3 \mathrm{~K} 4$ and $\mathrm{H} 3 \mathrm{~K} 27$.

To test whether JMJD3 is a histone demethylase, cDNA encoding for Myc-tagged JMJD3 was transfected into HeLa cells and the effect on histone lysine methylation was examined by immunocytochemistry using a specific antibody against trimethyl histone $\mathrm{H} 3$ lysine 27 (H3K27me3). Nucleus of each cell were visualized by DAPI staining and the expression of the exogenous JMJD3 was visualized by immunostaining against Myc. Ectopic expression of MycJMJD3 resulted in loss of $\mathrm{H} 3 \mathrm{~K} 27 \mathrm{me} 3$, in contrast to strong staining signals of $\mathrm{H} 3 \mathrm{~K} 27 \mathrm{me} 3$ in adjacent non-transfected cells (Figure 1A, top panel). To determine whether the loss of trimethylation is specific for the residue of histone $\mathrm{H} 3$ lysine 27, we also performed immunocytochemistry using specific antibodies against $\mathrm{H} 3 \mathrm{~K} 4 \mathrm{me} 3$ and $\mathrm{H} 3 \mathrm{~K} 9 \mathrm{me} 3$. Ectopic expression of Myc-JMJD3 had no effect on H3K4me3 and $\mathrm{H} 3 \mathrm{~K} 9 \mathrm{me} 3$, as the immunostaining signal intensities of $\mathrm{H} 3 \mathrm{~K} 4 \mathrm{me} 3$ and H3K9me3 did not change between transfected and non-transfected cells (Figure 1B). These data indicate that JMJD3 specifically targets H3K27 residue.

To examine whether JMJD3 is able to catalyze the removal of di- and monomethyl groups from H3K27, we also immunostained Myc-JMJD3 transfected cells with antibodies against these two histone modifications. Ectopic expression of Myc-JMJD3 had no effect on H3K27me2 and $\mathrm{H} 3 \mathrm{~K} 27 \mathrm{me} 1$, as the signal intensities of $\mathrm{H} 3 \mathrm{~K} 27 \mathrm{me} 2$ and H3K27me1 did not change between transfected and nontransfected cells (Figure 1A, middle and bottom panels).

Taken together, these data indicate that JMJD3 is likely a specific demethylase for $\mathrm{H} 3 \mathrm{~K} 27 \mathrm{me} 3$ in vivo.

The JmjC domain is required for histone demethylase activity

JMJD3 is a protein of 1682 amino acids. Conserved do-
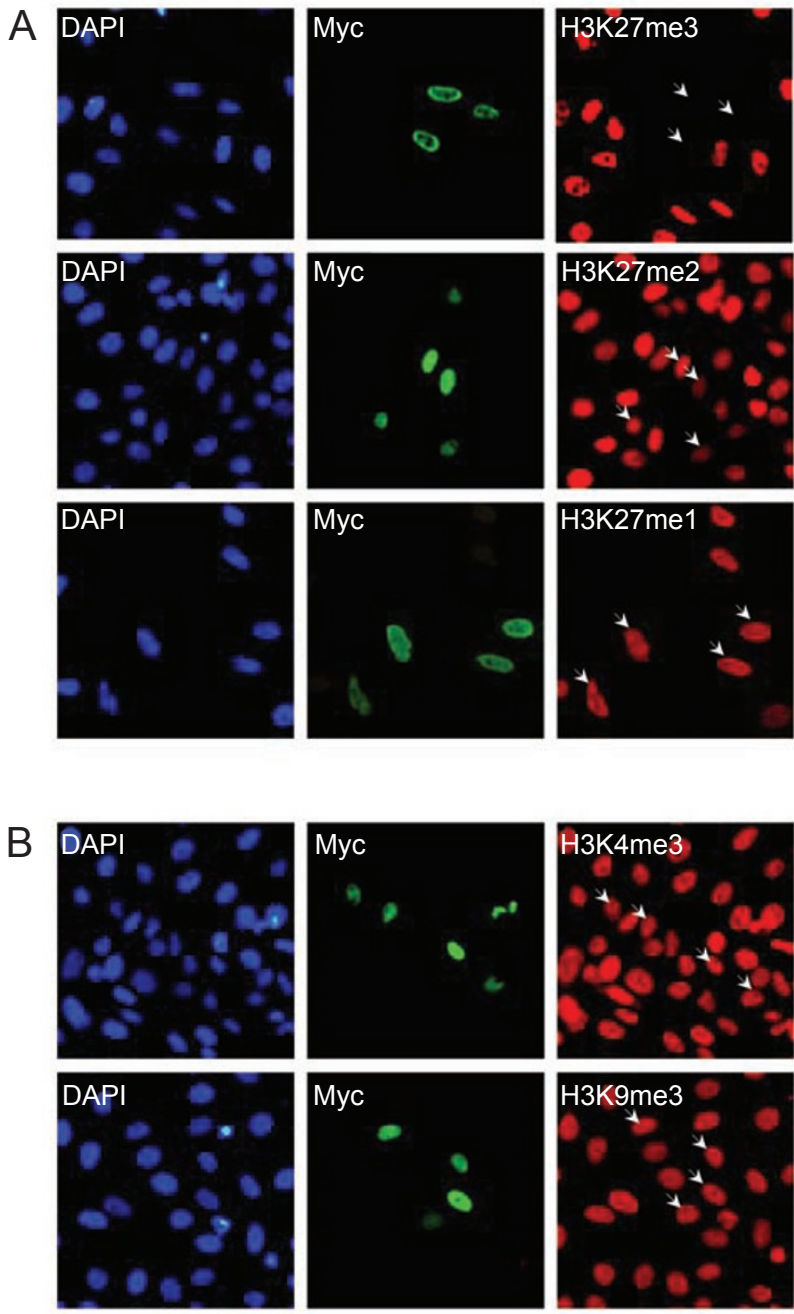

Figure 1 JMJD3 removes H3K27 methylation in vivo. HeLa cells transfected with Myc-JMJD3 were immunostained with specific antibodies against distinctively methylated lysine residues. Left panels are DAPI staining, middle panels are Myc staining, and panels on the right are methylated lysine staining (A, top: H3K27me3, middle: H3K27me2, bottom: H3K27me1; B, top: H3K4me3, bottom: H3K9me3). Arrows indicate Myc-JMJD3 expressed cells.

main analysis indicated that this protein contains only one recognizable conserved domain, the JmjC domain, at the $\mathrm{C}$-terminal of the protein. Crystal structure and biochemical studies have revealed that the JmjC domain is the catalytic center for histone demethylation [9, 25]. To determine whether the JmjC domain is required for the demethylase activity of JMJD3, we made a deletion mutant in which the JmjC domain was deleted (Figure 2A). Transfection of the deleted mutant into HeLa cells resulted in a truncated protein with an expected molecular weight (Figure 2B) and the transfected cells did not show reduction of the 
immunostaining signal of $\mathrm{H} 3 \mathrm{~K} 27 \mathrm{me} 3$, compared with the adjacent non-transfected cells (Figure 2C). These data indicate that the enzymatic activity of JMJD3 requires the JmjC domain.

Crystal structure of the JmjC domain of JMJD2A indicates that the catalytic domain is a jellyroll-like structure with eight $\beta$ sheets [25]. Three residues for chelating iron are conserved and required for the demethylase activity.
In JMJD3, these three residues are His1390, Glu1392, and His 1470. To determine whether this structure is required for the enzymatic activity, we mutated His 1390 to alanine (H1390A) (Figure 3A). Transfection of H1390A into HeLa cells produced a mutant protein with an expected molecular weight (Figure 3B) and the transfected cells maintained similar immunostaining signals of $\mathrm{H} 3 \mathrm{~K} 27 \mathrm{me} 3$ as the adjacent non-transfected cells (Figure 3C). These
A

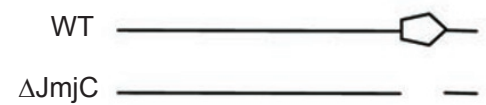

C

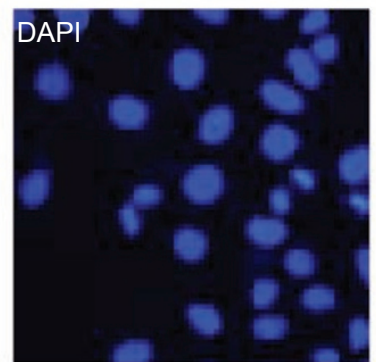

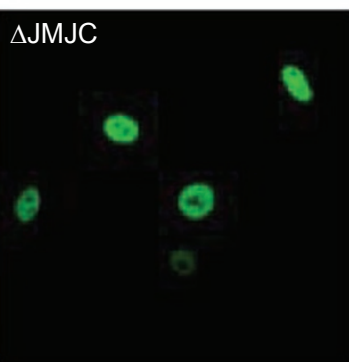

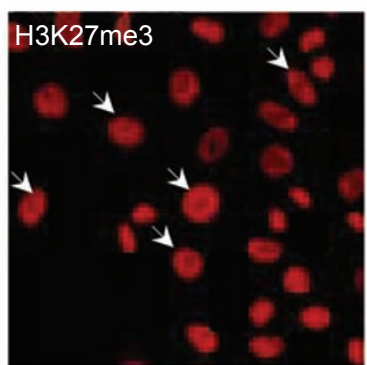

Figure 2 The JmjC domain is required for demethylase activity in vivo. (A) Schematic representation of the wild type and the JmjC domain deleted mutant. (B) HeLa cells transfected with the wild type and the JmjC domain deleted mutant were subjected to immunoblotting using an antibody against Myc. (C) HeLa cells transfected with the JmjC domain deleted mutant were immunostained with a specific antibody against H3K27me3. Left panel is DAPI staining, middle panel is Myc staining, and the panel on the right is $\mathrm{H} 3 \mathrm{~K} 27 \mathrm{me} 3$ staining. Arrows indicate cells expressing the deletion mutant.

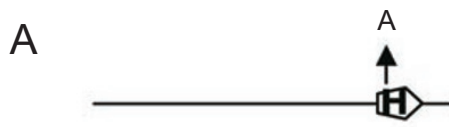

B

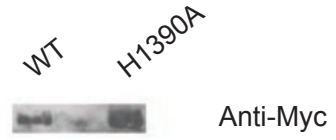

C
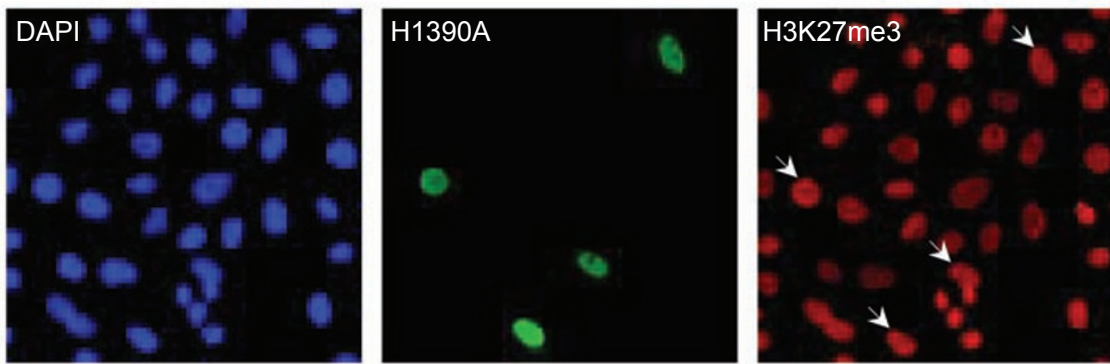

Figure 3 The conserved histidine H1390 is required for demethylase activity in vivo. (A) Schematic representation of the wildtype and H1390A mutant. (B) HeLa cells transfected with the wild type and the H1390A mutant were subjected to immunobloting using an antibody against Myc. (C) HeLa cells transfected with the H1390A mutant were immunostained with a specific antibody against $\mathrm{H} 3 \mathrm{~K} 27 \mathrm{me} 3$. Left panel is DAPI staining, middle panel is Myc staining, and the panel on the right is H3K27me 3 staining. Arrows indicate cells expressing the H1390A mutant. 
data indicate that JMJD3 is probably an iron-dependent dioxygenase, similar to other identified JmjC domain containing demethylases [25].

\section{JMJD3 demethylates $H 3 \mathrm{~K} 27$ in vitro}

To determine whether JMJD3 directly demethylates H3K27, we carried out a biochemical assay using bulk calf thymus histone proteins and GST-JmjC domain of JMJD3 that contains only the JmjC domain. The bulk calf thymus histone proteins used in the reaction contain a mixture of modified and non-modified histone H3. The methylation of histones can be visualized by western blot analysis using specific antibodies against distinctive methylated histones.

We first subcloned the C-terminal fragment of JMJD3 that contains the JmjC domain into a bacterial expression vector. The protein was fused to GST and the expression of the fusion protein was induced by IPTG. Through a glutathione-sepharose column, the fusion protein of the GST-JmjC domain of JMJD3 was purified as the expected size of $43 \mathrm{kDa}$ (Figure 4A). A $60-\mathrm{kDa}$ protein copurified with it was identified by mass spectrum analysis as GroEL, a chaperon in Escherichia coli. The purified fusion protein was incubated with bulk histones and the effect on lysine methylation was determined by western blot analysis. In the presence of various cofactors, the purified fusion protein was able to reduce the level of trimethyl H3K27 (Figure 4B), indicating that JMJD3 directly catalyzes the removal of H3K27me3. Surprisingly, the purified fusion protein was also able to reduce the level of dimethyl H3K27 and the demethylase activity toward dimethyl H3K27 was even stronger than to trimethyl H3K27 (Figure 4B). Furthermore, the purified fusion protein also reduced the level of monomethyl H3K27, even though to a much less extent (Figure 4B). These data suggest that other regions of the protein may participate in the determination of the substrate specificity.

To determine whether the demethylase activity of JMJD3 is specific to $\mathrm{H} 3 \mathrm{~K} 27$, we also carried out additional in vitro assays using the purified fusion protein of the GST-JmjC domain of JMJD3. Western blot analysis demonstrated that while a new batch of the purified protein could catalyze the removal of H3K27me3 (Figure 4C), it did not affect the levels of H3K9me3, H3K9me2, and H3K9me1 (Figure 4C). These data indicate that JMJD3 specifically demthylates $\mathrm{H} 3 \mathrm{~K} 27$.

\section{JMJD3 is upregulated in prostate cancer}

To test whether JMJD3 has a role in prostate cancer development and progression, we first searched the Oncomine database, a cancer profiling database containing more than 10000 microarrays [26]. Mining of the Oncomine database
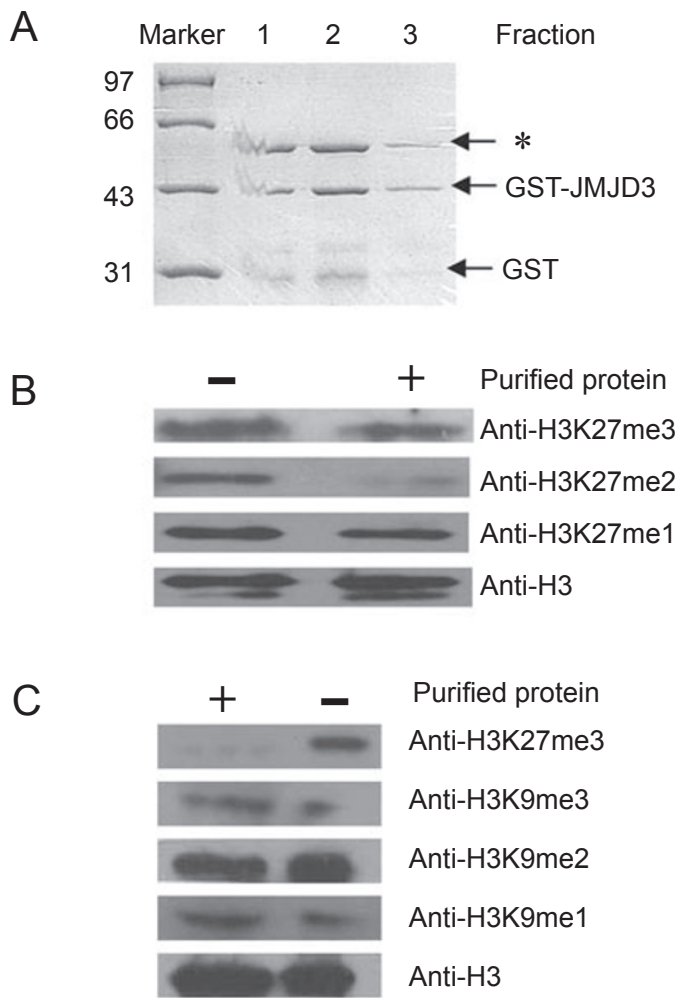

Figure 4 JMJD3 has demethylase activity in vitro. (A) A fusion protein of GST-JmjC domain of JMJD3 was expressed in E. coli and purified by a glutathione sepharose column. Various glutathione eluted fractions were subjected to Commassie blue staining. A $60 \mathrm{kDa}$ protein $(*)$ copurified was identified by mass spectrum analysis as GroEL, a chaperon in E. coli. (B and C) Histones were reacted with $(+)$ or without $(-)$ the purified fusion protein of GST-JmjC domain of JMJD3 in the presence of all cofactors and western blot analysis was performed using antibodies against tri-, di- and monomethyl H3K27 (B), tri-, di- and monomethyl H3K9 (C).

revealed that two sets of data from two different laboratories have expression profiles for JMJD3. In one set of data, the expression of JMJD3 was detected in only two out of 41 samples of benign prostate hyperplasia (4.8\%), but was detected in 10 out of 62 samples of prostate cancer $(16 \%)$, and five out of nine samples of metastatic prostate cancer $(55 \%)$ (Figure 5A). Average values of the expression indicate that JMJD3 expression is limited in benign prostate, but is upregulated in prostate cancer and the expression is even higher in metastatic disease (Figure 5B). This trend was observed in an independent set of data (Figure 5C).

To determine whether the protein levels of JMJD3 are also upregulated in prostate cancer, we performed western blot analysis in a number of frozen prostate tissues. Consistent with the microarray data deposited in Oncomine database, we could not detect JMJD3 protein in tissues of 
A

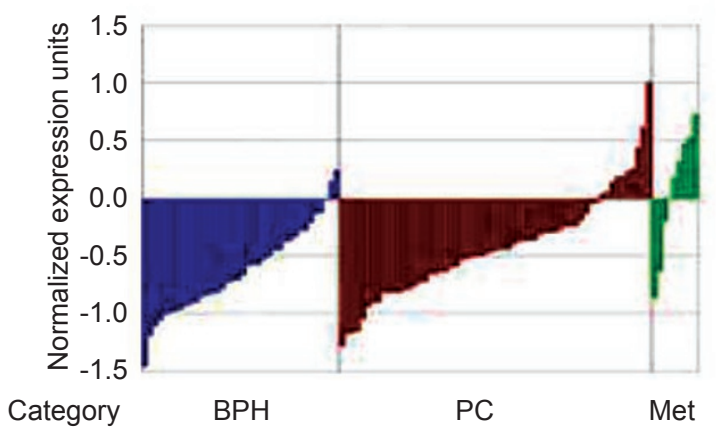

B

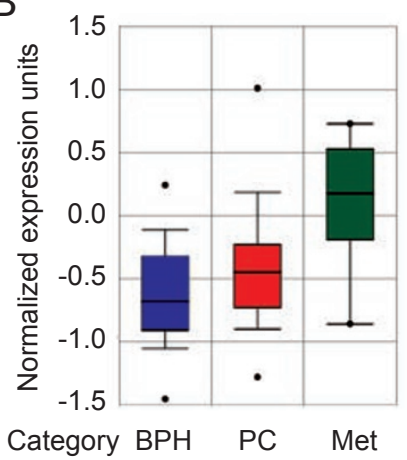

C

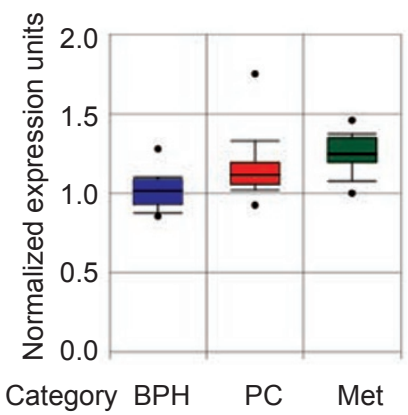

$\mathrm{D}$

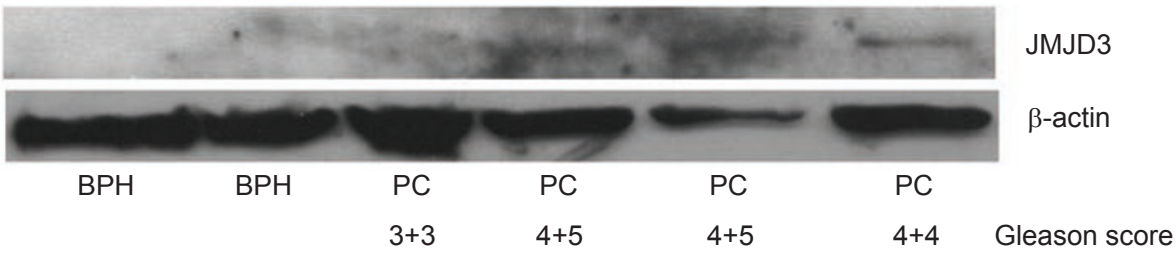

Figure 5 JMJD3 is upregulated in prostate cancer. (A-C) Gene expression data in Oncomine were analyzed. (A) The expression level of JMJD3 for each patient sample was shown. The patient numbers for each category are: $n=41$ for benign prostate hyperplasia (BPH), $n=62$ for prostate cancer (PC), and $n=9$ for metastatic prostate cancer (Met). ( $p$-value $=9.7 \mathrm{E}-5$ and correlation $=0.368$ ). (B) Average values of the JMJD3 expression for data set in (A) are shown. The thick bars in the boxes are average expression levels and the boxes represent $95 \%$ of the samples. The error bars are above or below the boxes, and the range of expression levels is enclosed by two dots. (C) Average values of the JMJD3 expression are shown for another set of microarray data. The patient numbers for each category are: $n=23$ for BPH, $n=64$ for PC, and $n=25$ for metastatic prostate cancer. ( $p$-value $=1.8 \mathrm{E}-10$ and correlation $=0.561$ ). (D) Western blot analysis of prostate tissue samples (BPH is benign prostate hyperplasia and PC is prostate cancer). The Gleason score for each patient was shown underneath.

benign prostate hyperplasia (Figure 5D), but JMJD3 was clearly detected in three out of four prostate cancer tissue samples. Pathological information indicates that one of the four prostate cancer tissues, in which JMJD3 protein was not detected in western blot analysis, has a Gleason score of $3+3$, as compared to other three tissues that have Gleason scores of at least $4+4$. Because higher Gleason score indicates more advanced cancer, the protein expression is thus consistent with the microarray data in which the expression levels of JMJD3 increase with disease severity.

\section{Discussion}

$\mathrm{N}$-terminal portion of histone $\mathrm{H} 3$ is a hot spot for histone lysine methylation. At least five lysine residues at the tail of histone $\mathrm{H} 3$ can be modified by methylation. These residues are lysine 4 (K4), K9, K27, K36, and K79. With the recent identification of lysine demethylases [6-16], methylation at $\mathrm{K} 4$, K9, and $\mathrm{K} 36$ were demonstrated to be reversible. However, whether methylation at $\mathrm{H} 3 \mathrm{~K} 27$ is reversible remained to be resolved. If methylation at $\mathrm{H} 3 \mathrm{~K} 27$ is reversible, what enzymes catalyze the demethylation?

In this paper, we demonstrated that methylation at
H3K27 is reversible and that JMJD3 catalyzes the removal of the trimethyl group from H3K27. These discoveries are consistent with a recent report that UTX and JMJD3 are histone H3K27 demethylases [27]. However, our finding that JMJD3 can only remove the trimethyl group, but not di- and monomethyl groups from $\mathrm{H} 3 \mathrm{~K} 27$, differs from the reported data in which JMJD3 can remove both tri- and dimethyl groups from $\mathrm{H} 3 \mathrm{~K} 27$ in vivo. The difference may result from the use of different expression vectors and, as a consequence, higher levels of the JMJD3 protein might be expressed in the study by Agger et al. [27]. Expansion of substrate specificity by a high concentration of histone demethylase has been observed for JMJD2A [10].

JMJD3 contains the JmjC domain at its C-terminus. Using the purified fragment containing the JmjC domain in the biochemical reaction in vitro, we demonstrated that JMJD3 directly catalyzes the removal of methylation from H3K27. However, this fragment can catalyze the removal of not only trimethyl group from H3K27 but also di-methylation of $\mathrm{H} 3 \mathrm{~K} 27$ with a higher activity, as well as monomethylation of H3K27. A longer fragment of JMJD3 only removed $\mathrm{H} 3 \mathrm{~K} 27 \mathrm{me} 3$, but not $\mathrm{H} 3 \mathrm{~K} 27 \mathrm{me} 2$ or $\mathrm{H} 3 \mathrm{~K} 27 \mathrm{me} 1$, in biochemical reactions in vitro [27]. These data suggest 
that other regions of the protein may be involved in the determination of the substrate specificity.

Since the discovery of the first histone demethylase, two of the identified histone demethylases have been shown to regulate the transcriptional function of the AR, a key molecule in prostate cancer development and progression [11, 17]. Also, two of them were also shown to be upregulated in prostate cancer [6, 24]. Furthermore, EZH2, a polycomb protein and a histone methyltransferase specific for $\mathrm{H} 3 \mathrm{~K} 27$, was shown to be upregulated in prostate cancer [21]. Together with the discovery that histone modifications can predict the prostate cancer recurrence, these data indicates that histone methylation at $\mathrm{H} 3 \mathrm{~K} 27$ may be tightly associated with prostate cancer development and progression. As the first step to determine whether JMJD3 is involved in prostate cancer, we examined the expression levels of JMJD3 in different stages of prostate cancer. Through mining of microarray data and western blot analysis on frozen tissue samples, we demonstrate that JMJD3 is upregulated in prostate cancer and the expression increases with disease progression.

It has been demonstrated that EZH2, a histone methyltransferase for $\mathrm{H} 3 \mathrm{~K} 27$, is also upregulated in prostate cancer [21]. Why are both histone methyltransferase and histone demethylase upregulated in prostate cancer? One possibility is that JMJD3 and EZH2 are upregulated in different prostate cancer tissues. Assuming that these proteins only have the respective biochemical functions as histone methyltransferase or demethylase, these data would suggest that completely opposite mechanisms are involved in different prostate cancer, which is consistent with the fact that prostate cancer is highly heterogeneous. Another possibility is that a compensation mechanism is adopted if both JMJD3 and EZH2 are upregulated in the same prostate cancer cells. When one gene is upregulated, H3K27 methylation is changed. As a compensation mechanism, the gene with the opposite biochemical function is also upregulated. This would suggest that prostate cancer cannot withstand broad alterations of H3K27 methylation. A third possibility is that upregulation of EZH2 in prostate cancer does not make use of the histone methyltransferase activity. This possibility is supported by the observation that other components required for the histone methyltransferase activity of EZH2 are not upregulated in prostate tissue [21], and is consistent with a recent study indicating that EZH2 has a transactivation activity separated from the SET-domain [28]. Future work would be directed to understand whether the methylation status of $\mathrm{H} 3 \mathrm{~K} 27$ is altered in prostate cancer and the roles of H3K27 methylation and JMJD3 in prostate cancer development and progression.

H3K27 methylation is a critical histone modification involved in X-chromosome inactivation [5]. It has been shown that methylation of $\mathrm{H} 3 \mathrm{~K} 27$ participates in embryonic development [29]. Since JMJD3 is a histone demethylase for $\mathrm{H} 3 \mathrm{~K} 27$, it would be interesting to determine whether this protein is involved in embryonic development by modulating H3K27 methylation.

\section{Acknowledgments}

We are grateful to Kazusa DNA Research Institute (Japan) for JMJD3 cDNA (KIAA0346) and to Dr Jenuwein (Research Institute of Molecular Pathology, Austria) for H3K27me3 antibody. This work was supported by Chinese Academy of Sciences (KSCX2-YW-R-04), National Basic Research Program of China (973 Program) (2007CB947900) and Shanghai Pujiang Plan (07pj14097).

\section{References}

1 Strahl BD, Allis CD. The language of covalent histone modifications. Nature 2000; 403:41-45.

2 Bannister AJ, Kouzarides T. Reversing histone methylation. Nature 2005; 436:1103-1106.

3 Bedford MT, Richard S. Arginine methylation an emerging regulator of protein function. Mol Cell 2005; 18:263-272.

4 Volpe TA, Kidner C, Hall IM, et al. Regulation of heterochromatic silencing and histone H3 lysine-9 methylation by RNAi. Science 2002; 297:1833-1837.

5 Plath K, Fang J, Mlynarczyk-Evans SK, et al. Role of histone H3 lysine 27 methylation in X inactivation. Science 2003; 300:131135.

6 Cloos PA, Christensen J, Agger K, et al. The putative oncogene GASC1 demethylates tri- and dimethylated lysine 9 on histone H3. Nature 2006; 442:307-311.

7 Klose RJ, Yamane K, Bae Y, et al. The transcriptional repressor JHDM3A demethylates trimethyl histone H3 lysine 9 and lysine 36. Nature 2006; 442:312-316.

8 Shi Y, Lan F, Matson C, et al. Histone demethylation mediated by the nuclear amine oxidase homolog LSD1. Cell 2004; 119:941953.

9 Tsukada Y, Fang J, Erdjument-Bromage H, et al. Histone demethylation by a family of JmjC domain-containing proteins. Nature 2006; 439:811-816.

10 Whetstine JR, Nottke A, Lan F, et al. Reversal of histone lysine trimethylation by the JMJD2 family of histone demethylases. Cell 2006; 125:467-481.

11 Yamane K, Toumazou C, Tsukada Y, et al. JHDM2A, a JmjCcontaining H3K9 demethylase, facilitates transcription activation by androgen receptor. Cell 2006; 125:483-495.

12 Christensen J, Agger K, Cloos PA, et al. RBP2 belongs to a family of demethylases, specific for tri-and dimethylated lysine 4 on histone 3. Cell 2007; 128:1063-1076.

13 Klose RJ, Yan Q, Tothova Z, et al. The retinoblastoma binding protein RBP2 is an H3K4 demethylase. Cell 2007; 128:889900.

14 Lee MG, Norman J, Shilatifard A, Shiekhattar R. Physical and functional association of a trimethyl $\mathrm{H} 3 \mathrm{~K} 4$ demethylase and 
Ring6a/MBLR, a polycomb-like protein. Cell 2007; 128:877887.

15 Yamane K, Tateishi K, Klose RJ, et al. PLU-1 is an H3K4 demethylase involved in transcriptional repression and breast cancer cell proliferation. Mol Cell 2007; 25:801-812.

16 Iwase S, Lan F, Bayliss P, et al. The X-linked mental retardation gene SMCX/JARID1C defines a family of histone H3 lysine 4 demethylases. Cell 2007; 128:1077-1088.

17 Metzger E, Wissmann M, Yin N, et al. LSD1 demethylates repressive histone marks to promote androgen-receptor-dependent transcription. Nature 2005; 437:436-439.

18 Shi YJ, Matson C, Lan F, et al. Regulation of LSD1 histone demethylase activity by its associated factors. Mol Cell 2005; 19:857-864.

19 Li X, Tsuji I, Kuwahara M, et al. Mass screening of prostate cancer in Changchun City of China. Int Urol Nephrol 2004; 36:541-548.

20 Seligson DB, Horvath S, Shi T, et al. Global histone modification patterns predict risk of prostate cancer recurrence. Nature 2005; 435:1262-1266.

21 Varambally S, Dhanasekaran SM, Zhou M, et al. The polycomb group protein EZH2 is involved in progression of prostate cancer. Nature 2002; 419:624-629.

22 Chen CD, Welsbie DS, Tran C, et al. Molecular determinants of resistance to antiandrogen therapy. Nat Med 2004; 10:33-39.

23 Klose RJ, Kallin EM, Zhang Y. JmjC-domain-containing proteins and histone demethylation. Nat Rev Genet 2006; 7:715-727.

24 Xiang Y, Chen C, et al. Unpublished data;

25 Chen Z, Zang J, Whetstine J, et al. Structural insights into histone demethylation by JMJD2 family members. Cell 2006; 125:691702.

26 Rhodes DR, Yu J, Shanker K, et al. ONCOMINE: a cancer microarray database and integrated data-mining platform. Neoplasia 2004; 6:1-6.

27 Agger K, Cloos PA, Christensen J, et al. UTX and JMJD3 are histone H3K27 demethylases involved in HOX gene regulation and development. Nature 2007; doi:10.1038/nature06147.

28 Shi B, Liang J, Yang X, et al. Integration of estrogen and Wnt signaling circuits by the polycomb group protein EZH2 in breast cancer cells. Mol Cell Biol 2007; 27:5105-5119.

29 Reik W. Stability and flexibility of epigenetic gene regulation in mammalian development. Nature 2007; 447:425-432. 